\title{
Emissivity measurements at room temperature on polymeric and inorganic samples
}

\author{
by F. Cernuschi' ${ }^{1}$ A. Russo ${ }^{2}$, G. M. Piana ${ }^{1}$, P. Mutti ${ }^{3}$, L. Viviani ${ }^{3}$ \\ 'ENEL S.p.A Centro di Ricerca Ambiente e Materiali, Via Rubattino, 5420134 Milano Italy; \\ ${ }^{2}$ Politecnico di Milano-Dipartimento di Energetica, P.zza L. da Vinci,32 20133 Milano Italy; \\ ${ }_{3}^{3}$ Politecnico di Milano- Dipartimento di Ingegneria Nucleare, Via Ponzio, 34/3 20131 Milano Italy
}

\begin{abstract}
An evaluation of the emissivity of polymeric and inorganic materials has been carried out in the spectral range $8-12 \mu \mathrm{m}$ using a direct technique. For polymeric samples the study focused on the dependence on the doping level. In the case of the inorganic samples measurements were aimed at the evaluation of the emissivity variations with the temperature Furthermore the effects on the emissivity of temperature differences between the sample and the background on the emissivity evaluation have been studied.
\end{abstract}

\section{Introduction}

Emissivity plays a key role in radiative heat transfer phenomena [1,2]; methodologies devoted to the evaluation of this parameter find applications in many industrial fields such as aerospace, optoelectronics, power generation, civil engineering, etc. Furthermore a knowledge of emissivity is fundamental for accurate temperature measurement using infrared detectors.

Direct and indirect measurement methods can be used. In the former case the emissivity is obtained by measuring the temperature of the sample, of a reference emitter applied to the sample and of the background. The latter is based on the measurement of the infrared reflectance from the sample surface; in this case the emissivity is computed as the complement to the reflectance.

\section{Experimental}

\subsection{Experimental set-up}

A sketch of the experimental set-up is shown in figure 1. The climatic chamber was designed and constructed to obtain a controlled, uniform temperature on the internal walls. It consists of a cubic box whose inner walls are covered by an aluminium layer painted black. The external ones were made of a suitable thermal insulator. Inside the chamber, a cooling coil reduced the background temperature and made it more uniform. The temperature distribution was monitored on-line by six Pt100 resistance thermometers placed on the inner surfaces of the chamber. A wide $10 \times 10 \mathrm{~cm}^{2}$ ) thermoelectric cooling-heating system guaranteed the temperature control of the samples (typically thin pellets of $12 \mathrm{~mm}$ diameter). An infrared mirror was placed close to the sample to measure the background temperature. In order to evaluate the sample temperature, half the surface was covered with 0.95 emissivity paint. An Inframetrics 600 infrared camera $(8-12 \mu \mathrm{m})$ was used to evaluate the apparent temperature of both the sample and the mirror. The emissivity values were then computed using the following expression $[3,4]$ :

$$
\varepsilon_{\mathrm{S}}=\frac{\left(\mathrm{T}_{\mathrm{S}}-\mathrm{T}_{\mathrm{BB}}\right)}{\left(\mathrm{T}_{\mathrm{BK}}-\mathrm{T}_{\mathrm{BB}}\right)} * \varepsilon_{\mathrm{BB}}
$$

where the subscripts $S, B B, B K$ refer to the sample, its black painted portion, and the background, respectively. 


\subsection{Materials}

Polymeric and inorganic samples have been studied. In the first case pressed pellets of polyaniline, polytiophene, and polypirrole powder with different doping levels were considered. A significant variation of the emissivity as a function of the doping level was expected.

\subsubsection{Polyaniline}

Commercial, highly doped polyaniline powder was obtained from Allied Signal inc. No information was available from the producer regarding the doping level or the type of dopant. Partial and complete undoping of the starting material was performed chemically using $\mathrm{NH}_{3}$.

\subsubsection{Polypirrole}

Doped polypirrole ( $30 \%$ mol.) was produced according to the methodology reported in [5]. Partial undoping was accomplished using $\mathrm{NH}_{3}$. No attempt was made to produce undoped material given the instability of polypirrole to spontaneous oxygen doping in air.

\subsubsection{Polythiophene}

Doped polythiophene powder $(30 \%$ mol.) was produced in accordance with the method described in [6]. Partial and complete undoping was achieved using $\mathrm{NH}_{3}$.

\subsection{4. $\mathrm{BaTiO}_{3}$}

In the case of inorganic materials $\mathrm{Sr}$ doped $\mathrm{BaTiO}_{3}$ pressed pellets were analyzed. A dependence of the emissivity on temperature was forecast due to the characteristic metalinsulator transition (MIT) for this material.

\section{Results and Discussion}

The emissivity values obtained for the polymeric samples at $20^{\circ} \mathrm{C}, 25^{\circ} \mathrm{C}$, and $30^{\circ} \mathrm{C}$ are reported In tables 1-3 respectively. Table 4 lists the results for the $\mathrm{BaTiO}_{3}$ sample (from $5^{\circ} \mathrm{C}$ to $90^{\circ} \mathrm{C}$ ). As far as $\mathrm{Sr}$-doped $\mathrm{BaTiO}_{3}$ is concerned, a simultaneous evaluation on a rough surface aluminium sample was carried out to discriminate an actual temperature dependance of the emissivity from spurious effects.

Conjugate polymer samples show a lower emissivity at higher doping levels. This is related to the increase in electrical conductivity and consequently higher IR reflectivity due to free carrier absorpion.

The fact that no emissivity variation was observed in $\mathrm{BaTiO}_{3}$ is due to the very low influence on reflectivity of the metal-insulator transition. In fact the MIT is associated with a variation of carrier mobility rather than an increase in carrier concentration; this, in tum, has a much smaller effect on the IR reflectivity.

The precision of the temperature value, the experimental configuration and statistical errors are the main causes of uncertainties in the emissivity measurements [7]. As far as the temperature values are concemed, their accuracy depends on the infrared camera features (characterized mainly by the Noise Equivalent Temperature Difference, the system bit resolution, the temperature range used and the non-linear response of the detector) which are well known. Thus, from their knowledge it is possible to estimate the magnitude of the uncertainties related to the temperature determination. As far as the present measurements are concerned, taking into account both statistical effects and the IR system features, the overall level of uncertainty works out (conservatively) to be about \pm 0.013 . On the other hand, uncertainties related to the experimental configuration (which minimised intelligent design and operation) cannot be quantified without a specific theoretical study, which is described in the following. 
Table 1

\begin{tabular}{|l|l|l|l|}
\hline Sample & $\begin{array}{l}\text { Sample } \\
\text { temperature } \\
{\left[{ }^{\circ} \mathrm{C}\right]}\end{array}$ & Emissivity & $\begin{array}{l}\text { climatic } \\
\text { chamber } \\
\text { average } \\
\text { temperature }\end{array}$ \\
\hline $\begin{array}{l}\text { completely } \\
\text { undoped }\end{array}$ & $20^{\circ} \mathrm{C}$ & 0.932 & $16.5^{\circ} \mathrm{C}$ \\
polytiophene & $25^{\circ} \mathrm{C}$ & 0.939 & \\
\hline partially & $20^{\circ} \mathrm{C}$ & 0.944 & \\
doped & $25^{\circ} \mathrm{C}$ & 0.906 & $16.5^{\circ} \mathrm{C}$ \\
polytiophene & $30^{\circ} \mathrm{C}$ & 0.917 & \\
\hline doped & $20^{\circ} \mathrm{C}$ & 0.921 & \\
polytiophene & $25^{\circ} \mathrm{C}$ & 0.760 & $16.5^{\circ} \mathrm{C}$ \\
& $30^{\circ} \mathrm{C}$ & 0.761 & \\
\hline
\end{tabular}

Table 2

\begin{tabular}{|l|l|l|l|}
\hline Sample & $\begin{array}{l}\text { Sample } \\
\text { temperature }\end{array}$ & Emissivity & $\begin{array}{l}\text { climatic } \\
\text { chamber } \\
\text { average } \\
\text { temperature }\end{array}$ \\
\hline partially & $20^{\circ} \mathrm{C}$ & 0.847 & $13.5^{\circ} \mathrm{C}$ \\
doped & $25^{\circ} \mathrm{C}$ & 0.851 & \\
polypirrole & $30^{\circ} \mathrm{C}$ & 0.857 & \\
doped & $20^{\circ} \mathrm{C}$ & 0.817 & $13.5^{\circ} \mathrm{C}$ \\
polypirrole & $25^{\circ} \mathrm{C}$ & 0.825 & \\
& $30^{\circ} \mathrm{C}$ & 0.824 & \\
\hline
\end{tabular}

Table 3

\begin{tabular}{|l|l|l|l|}
\hline Sample & $\begin{array}{l}\text { Sample } \\
\text { temperature }\end{array}$ & Emissivity & $\begin{array}{l}\text { climatic } \\
\text { chamber } \\
\text { average } \\
\text { temperature }\end{array}$ \\
\hline $\begin{array}{l}\text { completely } \\
\text { undoped }\end{array}$ & $20^{\circ} \mathrm{C}$ & 0.913 & $9.5^{\circ} \mathrm{C}$ \\
polyaniline & $35^{\circ} \mathrm{C}$ & 0.915 & \\
partially & $20^{\circ} \mathrm{C}$ & 0.916 & \\
doped & $25^{\circ} \mathrm{C}$ & 0.888 & $9.5^{\circ} \mathrm{C}$ \\
polyaniline & $30^{\circ} \mathrm{C}$ & 0.891 & \\
doped & $20^{\circ} \mathrm{C}$ & 0.889 & \\
polyaniline & $25^{\circ} \mathrm{C}$ & 0.725 & $9.5^{\circ} \mathrm{C}$ \\
& $30^{\circ} \mathrm{C}$ & 0.733 & \\
\hline
\end{tabular}

Table 4

\begin{tabular}{|c|c|c|}
\hline $\begin{array}{c}\text { Sample } \\
\text { temperature } \\
{\left[{ }^{\circ} \mathrm{C}\right]}\end{array}$ & $\begin{array}{c}\text { Emissivity } \\
\mathrm{BaTiO}_{3}\end{array}$ & $\begin{array}{l}\text { Emissivity } \\
\text { rough } \mathrm{Al}\end{array}$ \\
\hline 5 & 0.937 & 0.143 \\
\hline 10 & 0.938 & 0.140 \\
\hline 15 & 0.932 & 0.124 \\
\hline 20 & 0.950 & 0.792 \\
\hline 25 & 0.939 & 0.180 \\
\hline 30 & 0.942 & 0.178 \\
\hline 35 & 0.936 & 0.198 \\
\hline 40 & 0.938 & 0.168 \\
\hline 45 & 0.932 & 0.164 \\
\hline 50 & 0.932 & 0.163 \\
\hline 55 & 0.929 & 0.159 \\
\hline 65 & 0.929 & 0.163 \\
\hline 75 & 0.927 & 0.159 \\
\hline 85 & 0.925 & 0.163 \\
\hline 90 & 0.926 & 0.163 \\
\hline 75 & 0.928 & 0.162 \\
\hline 65 & 0.924 & 0.160 \\
\hline 55 & 0.931 & 0.160 \\
\hline 45 & 0.935 & 0.160 \\
\hline 35 & 0.934 & 0.161 \\
\hline 30 & 0.933 & 0.166 \\
\hline 25 & 0.944 & 0.166 \\
\hline 15 & 0.934 & 0.138 \\
\hline 10 & 0.936 & 0.144 \\
\hline 5 & 0.940 & 0.147 \\
\hline
\end{tabular}

In order to estimate the effects of the experimental sef-up on the emissivity values, emissivity measurements on a layer of polyalchitiophene and on a small aluminium plate with rough surface have been carried out in the range $5^{\circ} \mathrm{C}-70^{\circ} \mathrm{C}$. Polyalchitiophene was chosen for this experiment because as a polymeric material, it is able to tolerate temperature of the order of $70^{\circ} \mathrm{C}$ and since its emissivity is expected to be relatively insensitive to temperature variations. Small cyclic fluctuations of the emissivity as a function of the sample temperature have been observed, as is evident in figure 2. In order to explain this the relative position of mirror and sample has been taken into account.

As a matter of fact the sample and the mirtor are in two different positions so that they "see" (notwithstanding the use of a specific reciprocal alignment procedure) two different parts of the opposite wall side. In this way the background contributions on the surface of sample and mirror are different. This difference affects the precision of the emissivity measurement. An estimation of this effect can be made as follows: We have: 


$$
\begin{aligned}
& \mathrm{T}_{\mathrm{S}}=\varepsilon^{\text {true }} \mathrm{sT}_{\mathrm{S}}+\left(1-\varepsilon^{\text {true }} \mathrm{S}\right) \mathrm{T}_{\mathrm{B} 1} \\
& \mathrm{~T}_{\mathrm{BB}}=\varepsilon_{\mathrm{BB}} \mathrm{T}_{\mathrm{S}}+\left(1-\varepsilon_{\mathrm{BB}}\right) \mathrm{T}_{\mathrm{B} 1} \\
& \mathrm{~T}_{\mathrm{BK}}=\varepsilon_{\mathrm{MI}} \mathrm{T}_{\mathrm{MI}}+\left(1-\varepsilon_{\mathrm{MI}}\right) \mathrm{T}_{\mathrm{B} 2}
\end{aligned}
$$

where the subscript MI refers to mirror and the superscript "true" refers to the actual sample emissivity.

Substituting these expressions into equation (1), one obtains the following relation between the sample experimental emissivity value and the above-defined temperatures and emissivities:

$$
\varepsilon^{\text {meas }} \mathrm{s}=\frac{\varepsilon^{\text {true }} \mathrm{s}+\frac{\mathrm{x}}{\Delta \mathrm{T}}+\varepsilon_{\mathrm{MI}} \frac{\mathrm{T}_{\mathrm{B} 2}-\mathrm{T}_{\mathrm{MI}}}{\Delta \mathrm{T}}}{1+\frac{\mathrm{x}}{\varepsilon_{\mathrm{BB}} \Delta \mathrm{T}}+\frac{\varepsilon_{\mathrm{MI}}}{\varepsilon_{\mathrm{BB}}} \frac{\mathrm{T}_{\mathrm{B} 2}-\mathrm{T}_{\mathrm{MI}}}{\Delta \mathrm{T}}}
$$

where $x=T_{B 1}-T_{B 2}$ and $\Delta T=T_{S}-T_{B 1}$. Assuming $\varepsilon_{M}=0$, this reduces to:

$$
\varepsilon^{\text {meas }} \mathrm{S}=\frac{\varepsilon^{\text {true }} \mathrm{S}+\frac{\mathrm{x}}{\Delta \mathrm{T}}}{1+\frac{\mathrm{x}}{\varepsilon_{\mathrm{BB}} \Delta \mathrm{T}}}
$$

Eq. (4) shows that the discrepancy between measured and true sample emissivity increases in function of $x$ and decreases in function of $\Delta T$. The reason for this is that better results are generally obtained using the direct technique with a high temperature difference between sample and background and a very uniform background surrounding the sample and the mirror. Moreover the influence of these factors is stronger if the emissivity of the sample is low.

\section{Conclusion}

Partially satisfactory results are obtained on the polymeric samples where a dependence of the emissivity on doping level has been found. No significant variation of emissivity with temperature has been observed for the inorganic samples.

Concerning the error sources, very good agreement between a proposed theoretical model (which takes into account measurement conditions in estimating the uncertainty in measuring emissivity) and experimental data is obtained.

\section{REFERENCES}

[1] Incropera (F.P.) and DeWitt (D.P.) - Fundamental of heat and mass transfer. New York J. Wiley \& Sons, 1990

[2] Brewster (M.Q.) - Thermal Radiative Transfer Properties. New York, J. Wiley \& Sons, 1992

[3] Inframetrics - 600 operator's manual.

[4] NMAC - Infrared Thermography Workshop. EPRI NDE Center 6-8 August 1991

[5] Gregory (R.V.), Kimbrell (W.C.) and Kuhn (H.H.) - Conductive textiles. Synthetic Metals 28, $1989 \mathrm{C823}$ 
[6] Inoue (M. B.), Velazquez (E.F.) and Inoue (M.) - One-step chemical synthesis of doped polythiophene by use of copper (II) perchlorate as an oxidant. Synthetic Metals 24, 1988 pp. 223

[7] Hameury (J.) - Determination of uncertainties for emissivity measurements in temperature range $\left[200^{\circ} \mathrm{C}-900^{\circ} \mathrm{C}\right.$. Balageas (D.) Busse (G.), Carlomagno (G.M.) eds., Proc Quantitative Infrared Thermography 1994, Sorrento August 23-26, 1994, p.113-117.

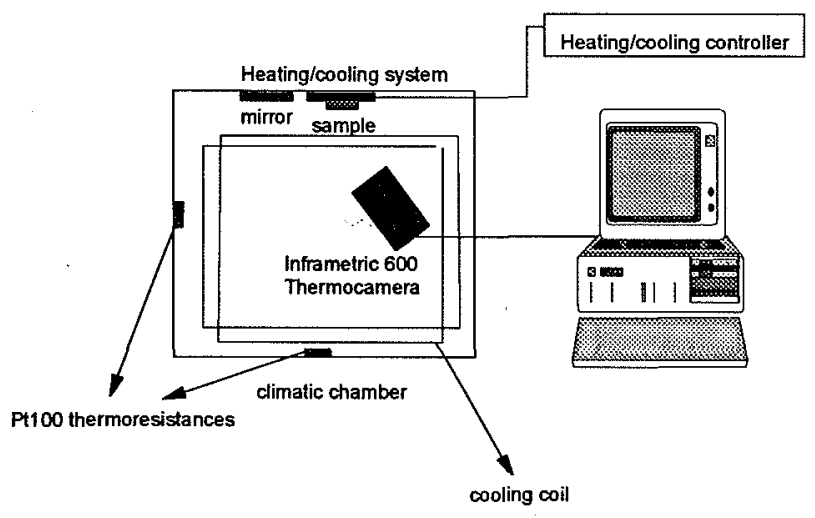

Fig. 1 Experimental set-up

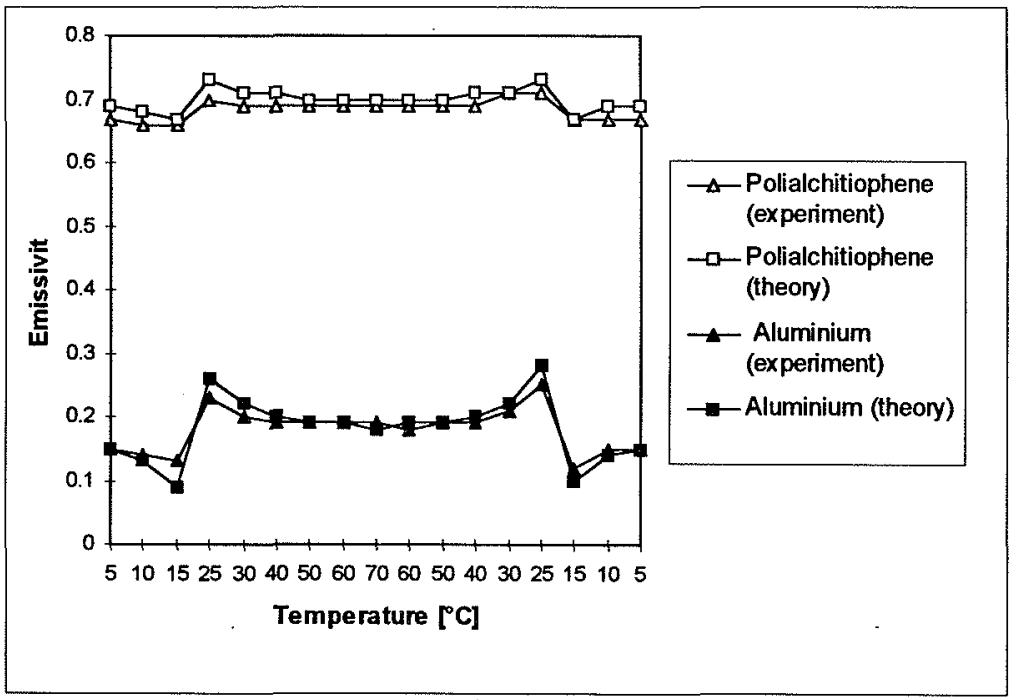

Fig. 2 Comparison between experimental data (for a polialchitiophene sample and an aluminium plate with rough surface) and the predicted effects of $\Delta T$ variations assuming an reasonable $x$ value of $1{ }^{\circ} \mathrm{C}$ taking as true emissivity the value measured at the highest temperature. 